\title{
Human Bite on Nose
}

\author{
Prasit Biswas, ${ }^{1}$ Shriyash Sinha, ${ }^{1}$ Somnath Saha ${ }^{1}$
}

\begin{abstract}
Introduction
Human bites are notorious due to chance of infection by direct inoculation of pathogens from saliva and must be managed properly. Human bite injuries cause dilemma to the treating doctor regarding its way of management. The options of management are primary closure, delayed closure with skin/tissue grafting and conservative.

Case Report

A 43 year old male patient with a human bite injury with tissue loss on the tip of nose was admitted in hemodynamically stable condition. He was given tetanus toxoid and anti-rabies vaccination as per current guidelines. Delayed wound closure was done in 2 steps with forehead flap, in 3 weeks interval. Patient was discharged after suture removal. There was good color matching and no complications.

$\underline{\text { Discussion }}$

Human bite injuries are mostly due to inter personal violence, alcohol intoxication and psychiatric illnesses. Multiple instances of such bite injuries have been reported. Delayed closure of bite injuries with forehead flap provides good colour and texture match as well as good flap survival due to rich vascularity.
\end{abstract}

ABSTRACT

$\underline{\text { Kevwords }}$

Bites, Human; Nose; Rhinoplasty; Forehead; Surgical Flaps

$\mathrm{H}$ uman bites of the face present as a surgical challenge, sometimes with a dilemma as to the method and timing of surgical repair. Primary surgical repair is the treatment of choice for most clinically uninfected facial bite wounds, whereas delayed closure should be reserved for certain high risk or infected wounds, and especially when there is partial or total loss of important structure(s) of the face. Avulsed injuries with significant tissue loss represent the most difficult cases for definitive management. Reconstruction becomes absolutely necessary in such patients to avoid permanent cosmetic defect. The cosmetic effects of such losses are profound and may affect the social life and even sometimes the livelihood of the individual patient involved. Many of the patients who sustain human bite of the face present with healed wound but with disfiguring scars and deformed facial appendages also needing reconstruction.

Human bites can be classified depending on the mechanism of injury into, occlusion bites and the closed fist bite (or fight bite). ${ }^{1}$ Occlusion bites occur when the teeth are sunk into the skin with sufficient force to breach the integrity of the skin. Clenched fist injuries occur when a closed fist impacts another individual's teeth, leaving an injury over the dorsal aspect of the third, fourth or fifth metacarpophalangeal (MCP) joints, most classically over the third MCP. ${ }^{2}$ Direct occlusion bites manifest a more or less distinct impression of the biter's teeth. A bite by an adult can usually be reliably distinguished from one by a child (smaller radius, impressions of individual teeth, deciduous dentition). A distance greater than $3 \mathrm{~cm}$ between canine teeth indicates that the bite wound was inflicted by an adult. Bite wounds caused by children who still have their primary dentition are characterized by a distance between molars that is usually less than $2.5 \mathrm{~cm}^{3}$

\section{Case Report}

A 43 year old male presented to the ENT OPD with a 4 day old human bite post inter-personal violence. He was systematically fine and had no other complaints. The bite wound was circular, about $2 \mathrm{~cm}$ in diameter, situated at

1 - Department of ENT, Calcutta National Medical College

Corresponding author:

Dr Prasit Biswas

email: prasit.biswas93@gmail.com 


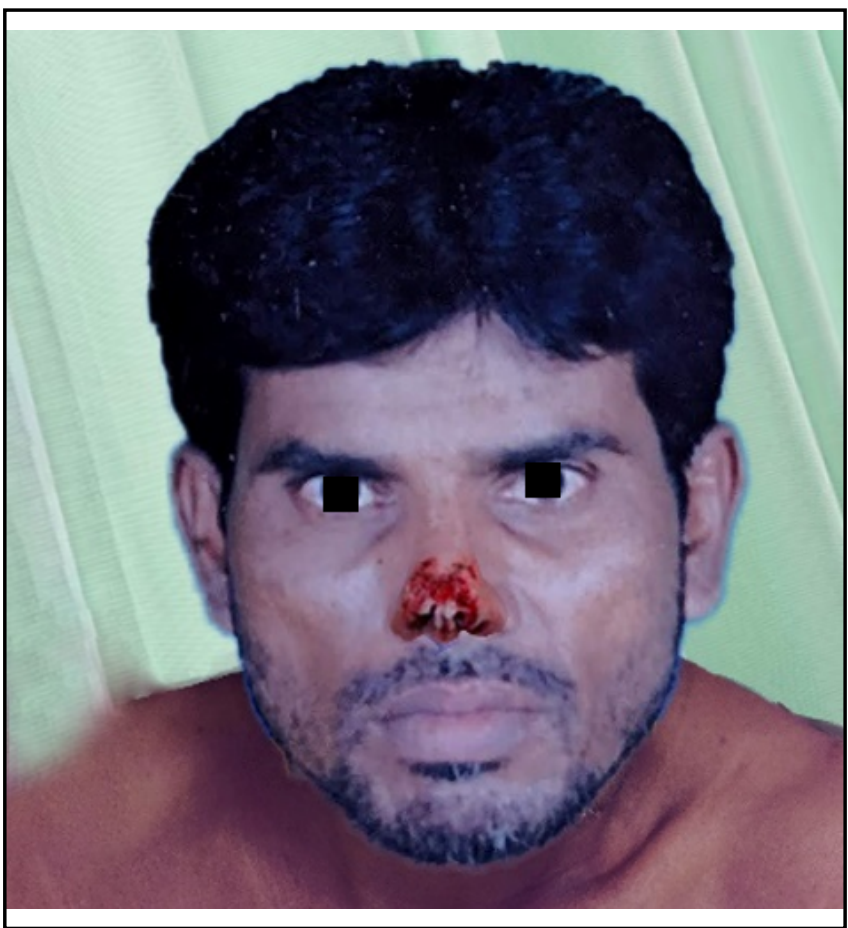

Fig. 1. Preoperative photo of the wound

the tip of nose with loss of skin, subcutaneous tissue and also loss of medial crus of alar cartilage and small part of caudal end of nasal septum.

The wound was not infected, without any discharge or active bleeding. Initial wound care, dressing, was given by his primary health centre. Primary closure was not possible due to the loss of tissue and possibility of poor cosmetic outcome. The patient was admitted with plan of nasal reconstruction using forehead flap. Thorough wound cleaning and fresh dressing were done after admission. The patient had already received Injection Tetanus toxoid. Anti-rabies vaccination and Rabies immunoglobulin were given as per recommended schedule. ${ }^{4}$ Routine investigations, serology was done and pre-anaesthetic fitness was taken. Reconstruction was done by rotating forehead flap from right side. The bare area on the patient's forehead was covered with a split thickness skin graft taken from his right thigh (Fig.2) Patient was put on iv antibiotics post operatively.

Twenty one days after the initial reconstruction, patient was again taken for surgery. The flap was repositioned and sutured with 4-0 Nylon. (Fig. 3)

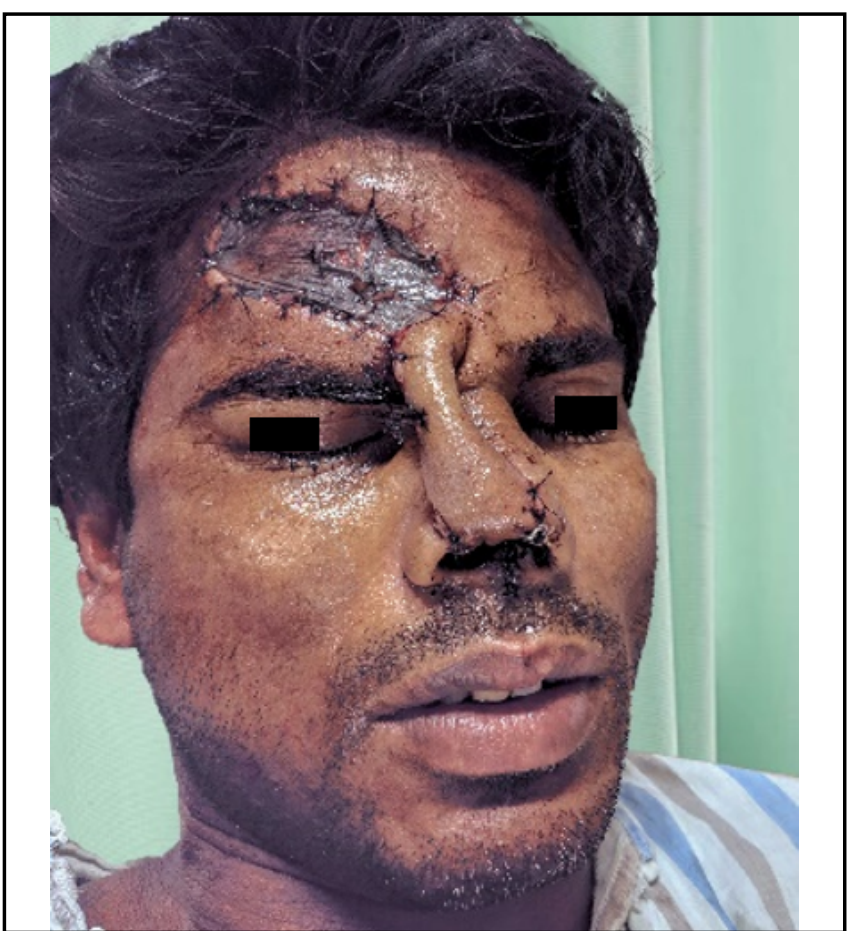

Fig. 2. Reconstruction was done by forehead flap

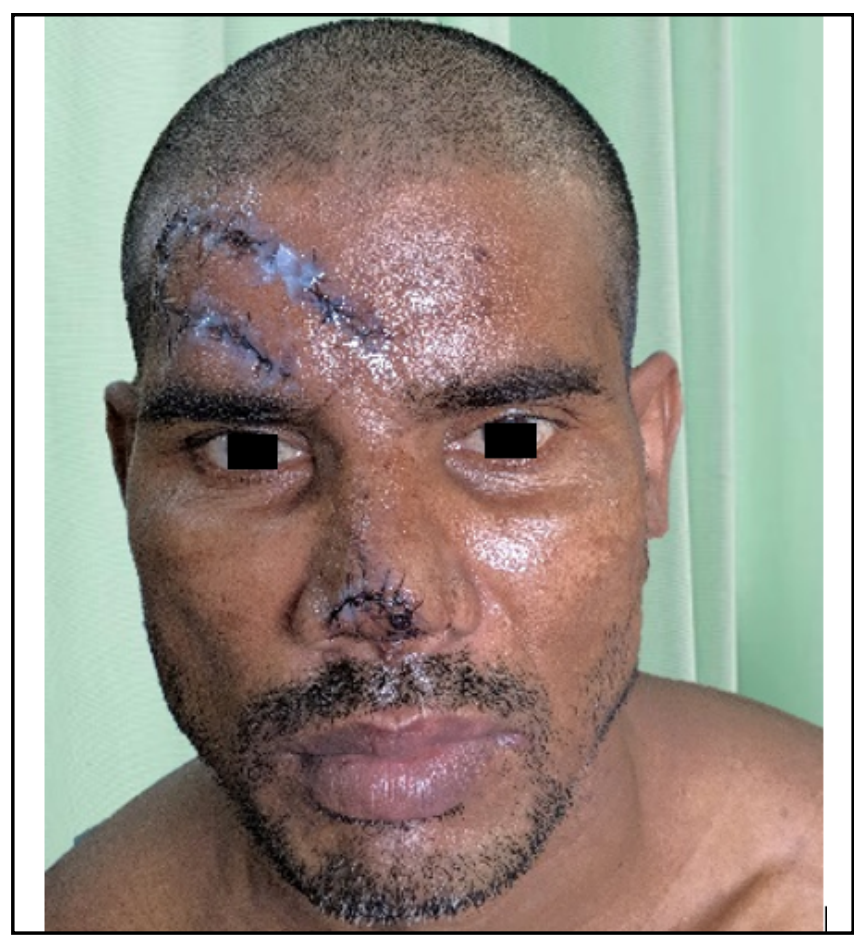

Fig.3. Clinical photograph after repositioning the flap 


\section{Discussion}

Human bites are serious injuries that may result in infection, loss of function and gross disfigurement.

A classification of the severity of bite wounds, from Rueff et $\mathrm{a}^{5}$

Grade I

- Superficial skin lesion

- Torn skin

- $\quad$ Scratched skin

- $\quad$ Bite canal

- Crushing injury

Grade II

- Wound extending from the skin to the fascia, muscle, or cartilage

Grade III

- Wound with tissue necrosis or tissue loss

Infection from oral contaminants, tissue damage, and difficult surgical reconstruction make the management of human bite injuries a challenge. Rate of infection in different bite injuries are as follows: ${ }^{6}$ Cat bites: $30-50 \%$, Human bites: $15-25 \%$, Dog bites:5-25\% Overall: 1020\%, Hand: $18-36 \%$, Arm: $17-20 \%$, Leg: 7-15\%,Face: $4-11 \%$. Tetanus toxoid is essential after any bite injury. Although rare, Rabies can be transmitted via human bite also. Anti-rabies vaccination is advisable as per WHO recommended ARV schedule. ${ }^{4}$

- The 5 dose intramuscular regime (1-1-1-1-1) : one dose of vaccine to be administered on days 0,3 , $7,14 \& 28$ in the deltoid region for adults and anterolateral aspect of thigh in children.

- Dose of rabies immunoglobulin (RIG): $20 I U / \mathrm{kg}$ of Human RIG or $40 \mathrm{IU} / \mathrm{kg}$ of Equine RIG. Administration of RIG can be delayed till 7 days from the date of first vaccine dose.

A recent incident published in Times of India reports of a mentally challenged man, biting 22 pedestrians at Howrah, West Bengal. ${ }^{7}$

Another incident published in Times of India reports of an intoxicated man biting a snake into pieces after getting bitten by it. ${ }^{8}$

The main reasons of sustaining human bites are interpersonal violence and physical assault, mental instability, intoxication.

The goals of reconstructive surgery is to achieve full wound closure, restoration of normal anatomical landmarks and leaving behind least chance of scarring and healing by secondary intention. Study conducted by Saha et. al. found that forehead flap is one of the safest cutaneous flaps available in reconstructive surgery of nose and orofacial region. It provides good colour and texture match and is quick and easy to raise as well as good flap survival due to rich vascularity. ${ }^{9}$

\section{References}

1. Conlon HA. Human bites in the classroom: incidence, treatment, and complications. J Sch Nurs. 2007 Aug;23(4):197-201

2. Fight-Bite: Not Just a Hand Problem. Cook CP, Knox MJ, Wimberly RL, Ellis HB, Riccio AI. J Pediatr Orthop. 2014; 34(6):647-9. doi: 10.1097/BPO.0000000000000155

3. Herrmann B, Dettmeyer R, Banaschak S, Thyen U. Typische Bissverletzungen als Blickdiagnose. In: Herrmann B, Dettmeyer R, Banaschak S, Thyen U, eds. Kindesmisshandlung. Heidelberg: Springer; 2008. 58 pp. https://link.springer.com/bo ok/10.1007\%2F978-3-662-48844-7

4. WHO recommendation for ARV prophylaxis. https://www. who.int/ith/vaccines/rabies/en/

5. Rueff F, Bedacht R, Schury G. [Bite injury. Special situation as to clinical aspects, therapy and course of healing]. Med Welt. $\quad 1967 ; \quad 12: 663-8 . \quad$ https://www.ncbi.nlm.nih.gov/ pubmed/5586321/

6. Brook I. Management of human and animal bite wound infection: an overview. Curr Infect Dis Rep. 2009;11(5):38995.

7. Banerjee R.. Howrah : mentally challenged man bites 22 , run amok on GT road, Times of India 2019, July 28

8. ANI. Uttar Pradesh: Drunk UP man bites snake into pieces after it bit him, Times of India 2019, July 29

9. Saha VP, Ghosh A, Kesarwaani S, Saha S.(2019). Forehead flap for reconstruction of orofacial defects : A tertiary care centre experience, West Bengal, India. Indian Journal of Applied Research 2019; 9(6), 51-3. 